\title{
Investigation of amorphization energies for heavy ion implants into silicon carbide at depths far beyond the projected ranges
}

\author{
E. Friedland* \\ Physics Department, University of Pretoria, 0002 Pretoria, South Africa
}

Keywords: Silicon carbide, ion implantations, amorphization energies.

*E- mail: erich.friedland@up.ac.za, Phone: +27-12-4202453, Fax: +27-12-3625288

\begin{abstract}
At ion energies with inelastic stopping powers less than a few $\mathrm{keV} / \mathrm{nm}$, radiation damage is thought to be due to atomic displacements by elastic collisions only. However, it is well known that inelastic processes and non-linear effects due to defect interaction within collision cascades can significantly increase or decrease damage efficiencies. The importance of these processes changes significantly along the ion trajectory and becomes negligible at some distance beyond the projected range, where damage is mainly caused by slowly moving secondary recoils. Hence, in this region amorphization energies should become independent of the ion type and only reflect the properties of the target lattice. To investigate this, damage profiles were obtained from $\alpha$-particle channeling spectra of $6 \mathrm{H}$ $\mathrm{SiC}$ wafers implanted at room temperature with ions in the mass range $84 \leq M \leq 133$, employing the computer code DICADA. An average amorphization dose of $(0.7 \pm 0.2)$ dpa and critical damage energy of $(17 \pm 6) \mathrm{eV} /$ atom are obtained from TRIM simulations at the experimentally observed boundary positions of the amorphous zones.
\end{abstract}

\section{Introduction}

Silicon carbide is an important ceramic material with many technological applications ranging from cladding material of nuclear fuel elements to electronic devices in high-temperature and harsh radiation environments. Its thermal stability and especially its amorphization properties during ion implantation have therefore extensively been investigated. Although irradiation-induced amorphization at typical ion implantation energies is primarily caused by displacements during ballistic collisions, results also point to an influence of inelastic processes on damage creation [1,2]. A direct determination of their importance was obtained from an analysis of critical damage energies involved in the production of buried graphitized layers in diamond by $\mathrm{C}^{+}$ions [3-5]. It revealed a significant defect annealing effect by electronic stopping, leading to strongly reduced damage efficiencies. However, a similar investigation of damage creation in silicon carbide showed the opposite effect. In this case damage efficiency is strongly enhanced by electronic stopping, leading to lower amorphization energies [6], which might be explained by a reduction of binding energies due to electronic excitation or ionization of target atoms. A comparative study showed that in monoelemental targets the annealing effect generally dominates, while in binary targets both opposing effects are important, leading either to an enhanced or reduced damage efficiency depending on implantation parameters [7]. In silicon carbide, enhanced damage efficiency is generally observed with increasing electronic stopping $[6,8]$. However, the importance of the inelastic processes reduces significantly along the ion trajectory, as it depends not only on the ion's mass but also on its velocity. Furthermore, at some distance beyond the projected range ion properties become unimportant, as ions and primary knockon atoms do not reach this depth and damage is only caused by slowly moving secondary recoils. 


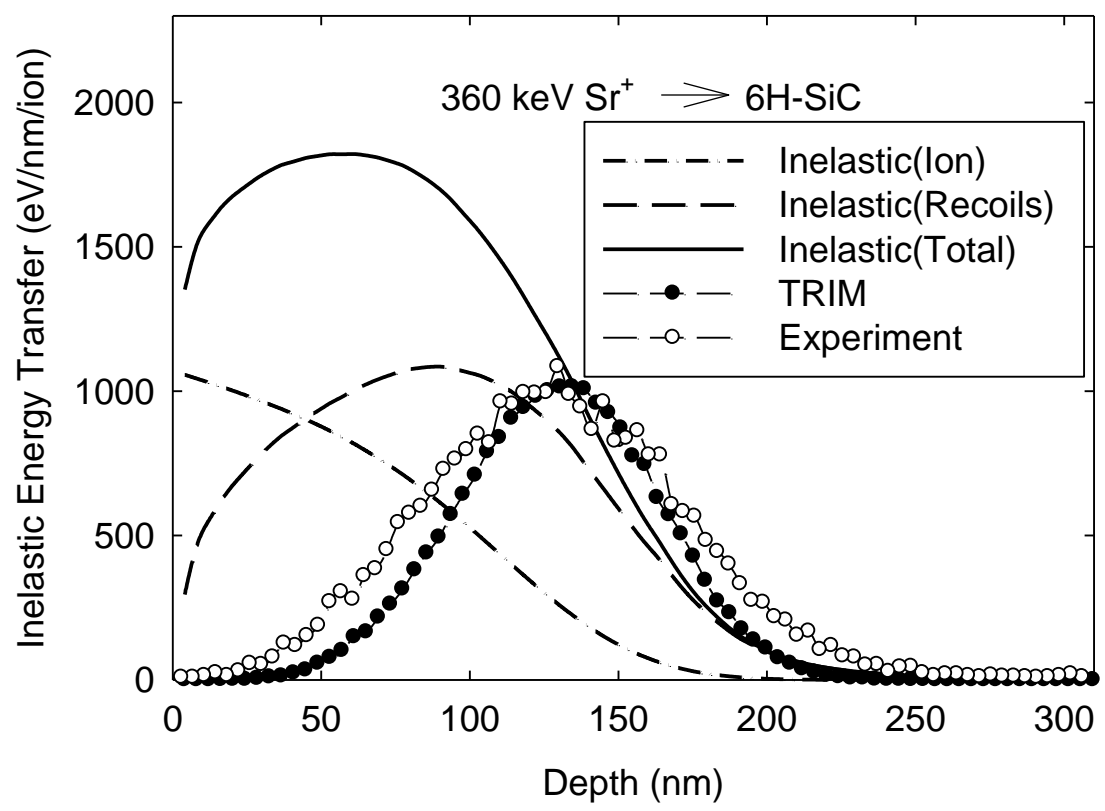

Fig. 1: TRIM-simulation of inelastic energy transfers for $360 \mathrm{keV} \mathrm{Sr}{ }^{+}$ions in $6 \mathrm{H}-\mathrm{SiC}$ together with the calculated and experimentally obtained implantation profiles in relative units.

Fig. 1 illustrates this situation for the case of strontium ions implanted at $360 \mathrm{keV}$, using version 98.01 of the well-known pseudo Monte Carlo simulation TRIM [9]. This code employs a universal atomic potential and makes use of statistical distributions of free path lengths and collision parameters for amorphous matter, which makes it much faster than a proper Monte Carlo simulation. High computing speed was essential for this investigation, as about $10^{5}$ ion trajectories had to be simulated using the full-cascade option to obtain the desired statistical certainty at the appropriate depths. The inelastic energy transfers due to the ion and recoiling atoms are shown together with the simulated and experimental implantation profiles in relative units.

Up to a depth of the projected range at $130 \mathrm{~nm}$ the total inelastic energy density is relatively high and can significantly influence damage production by the two competing processes of binding energy reduction and defect annealing. The first process, due to the electronic excitation of lattice atoms, causes an increased atomic displacement rate and therefore a lower amorphization dose, while the opposite process of damage annealing will increase the amorphization dose. Damage annealing at inelastic energy densities encountered in this investigation is probably only due to irradiation-induced defect mobility. The effect of heat spikes, which can significantly increase damage recovery, is only expected at inelastic energy densities above approximately $5 \mathrm{keV} / \mathrm{nm}[10]$.

At a depth of approximately twice the projected range the contributions from the ion is zero and even those of the recoils becomes small. Contrary to the surface region, where heterogeneous amorphization is observed due to the occurrence of high-defect density islands produced in collision cascades [11], progressive defect accumulation by low-energy elastic collisions, not influenced by inelastic processes or non-linear effects, is expected in this region. This should cause homogeneous amorphization after a critical defect density is reached.

\section{Experiment and analysis}

To obtain amorphization up to depths of approximately twice the projected range, fluences of the order of $10^{16} \mathrm{~cm}^{-2}$ of $\mathrm{Kr}, \mathrm{Sr}, \mathrm{Ag}, \mathrm{I}, \mathrm{Xe}$ and $\mathrm{Cs}$ ions are implanted at room temperature with an energy of $360 \mathrm{keV}$ in hexagonal $6 \mathrm{H}-\mathrm{SiC}$ wafers from Intrinsic Semiconductors ${ }^{\circledR}$. Typical random and aligned backscattering spectra along the (0001) direction are presented in Fig. 2 for the strontium 


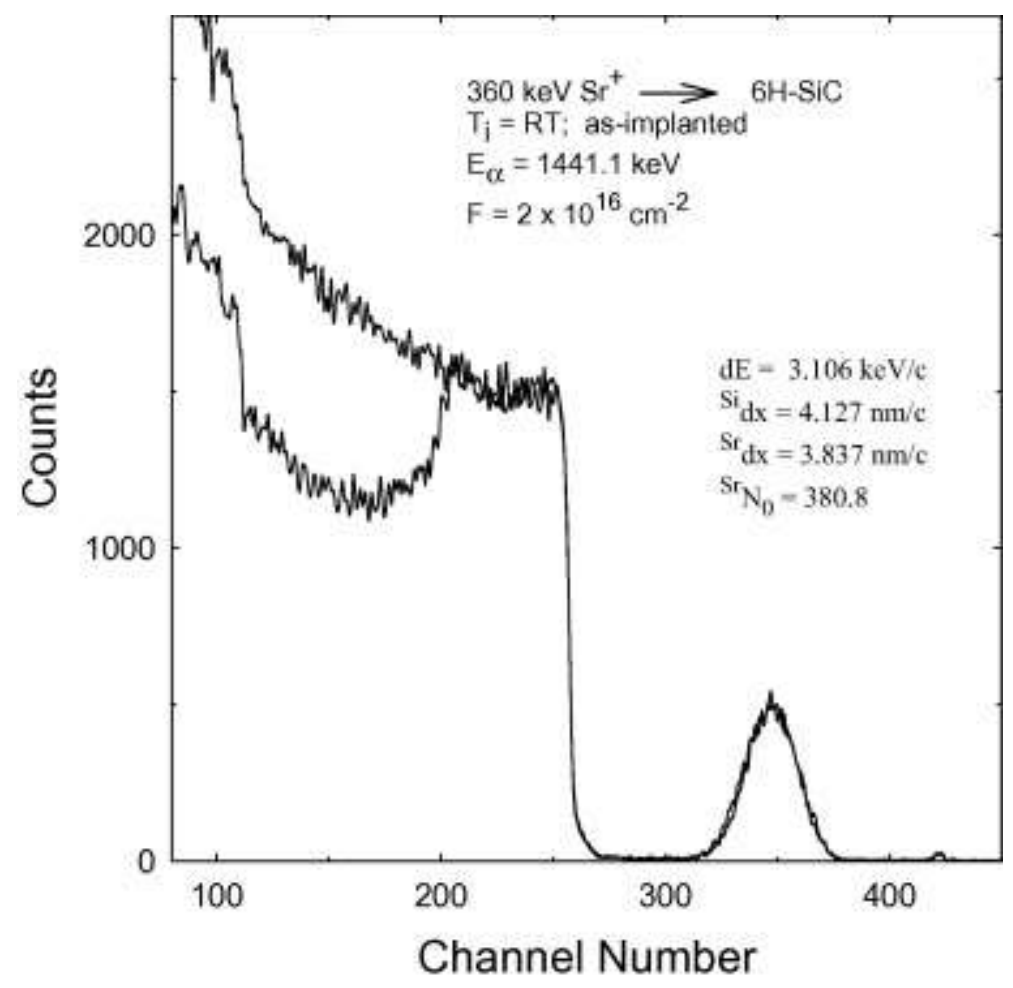

Fig. 2: Random and aligned backscattering spectra of $6 \mathrm{H}-\mathrm{SiC}$ implanted with $360 \mathrm{keV} \mathrm{Sr}{ }^{+}$at room temperature with a fluence of $2 \times 10^{16} \mathrm{~cm}^{-2}$. The first three numbers in the inset are the energy resolution and the depth resolutions for silicon and strontium per channel, while the fourth is the channel number of the strontium surface.

implant, showing an amorphous region extending from the surface to a depth of $227 \mathrm{~nm}$ at a projected range of $130 \mathrm{~nm}$. The integrated yield of the implanted ion species agreed within experimental uncertainty in all cases with the nominal fluence, which is used in all calculations. More details of the experimental method used are reported elsewhere [12]. Damage profiles where obtained from the channeling spectra using the computer code DICADA [13], which makes use of a modified master equation approach based on the channeling concept of the Lindhard theory. The latter theory under-estimates the yield of the aligned spectrum because of the assumption that conservation of transverse energy in an ideal crystal holds right from the surface, while this is only true after the ions have penetrated a relatively large number of atomic layers. This is rectified in the modified master equation by using start values for the minimum yield at the surface obtained from a full Monte Carlo simulation. DICADA calculations can be applied to compounds containing different kinds of point defect configurations, including clusters and amorphous zones, but do not take into account stacking faults and dislocation loops, which have relatively large de-channeling cross sections, leading to artificial tail-effects at larger depths. Assuming randomly displaced atoms, this effect was observed in all samples. To include extended defects, a modified version is used, which originally had been developed for mono-elemental targets only $[14,15]$. For the current calculations a Debye temperature of $\theta_{D}=1030 \mathrm{~K}$ is used, which is an average of the widely varying published values [16]. Furthermore lattice parameters of $a=0.3081 \mathrm{~nm}, c=1.5092 \mathrm{~nm}$ and an atomic density of $\rho=9.662 \times 10^{22}$ at $\mathrm{cm}^{-3}$ were assumed. Relative damage densities of the total damage, not corrected for de-channeling effects of extended defects, and of randomly displaced atoms are shown in Fig. 3 for the strontium implant. The position of the amorphous-crystalline $(\mathrm{a} / \mathrm{c})$ interface is taken as the depth where the relative defect density dropped significantly below unity. 


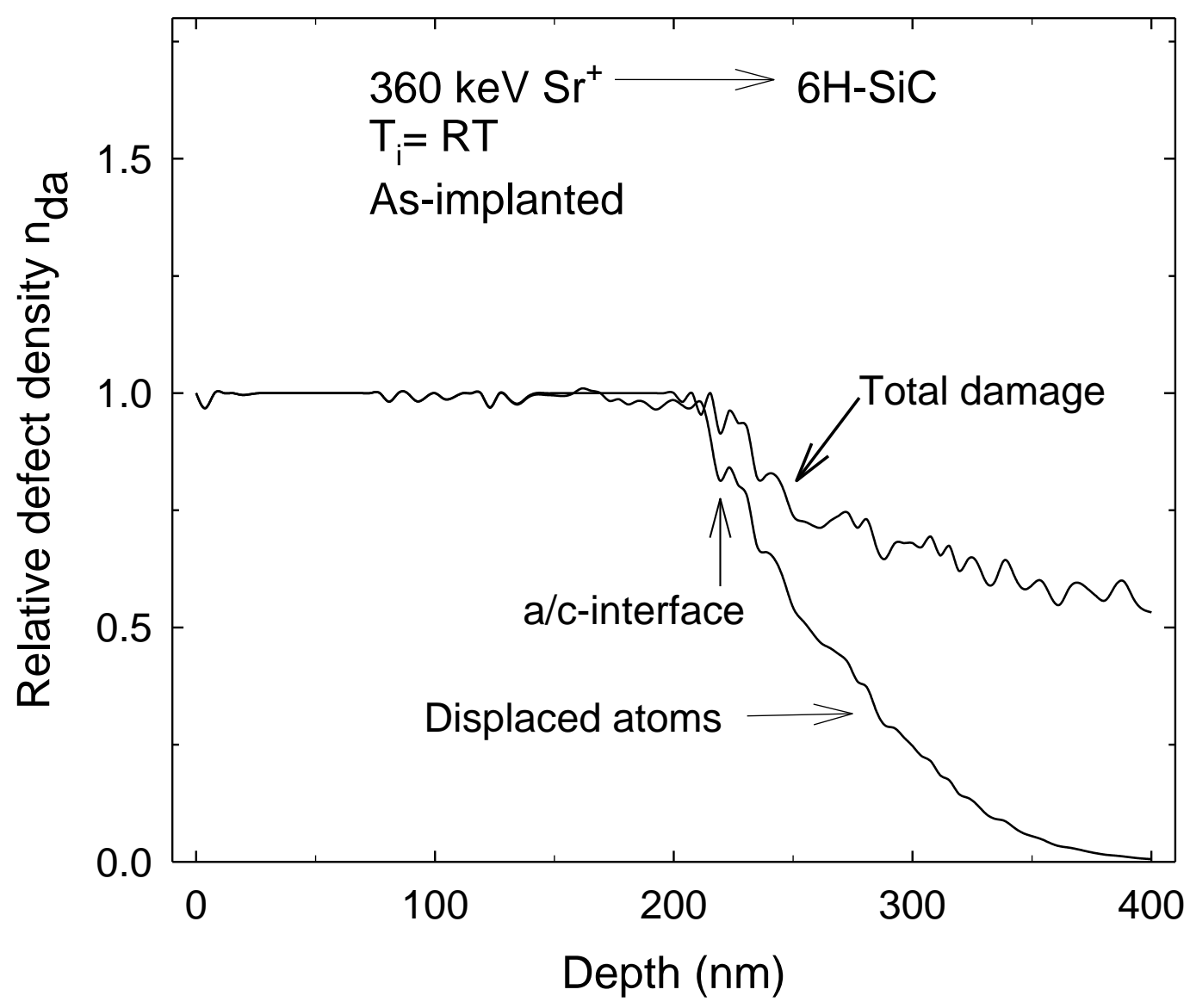

Fig 3: Relative defect density distributions for the total damage (not corrected for de-channeling effects of extended defects) and for randomly displaced atoms, obtained from the spectra of fig. 2 using the DICADA code.

Fig. 4 shows the TRIM estimates of elastic energy transfers to the target lattice with a depth scale slightly adjusted, to correct for the difference of the simulated and experimentally obtained projected ranges. A depth correction factor $f=1.019$ is used in the case of the strontium implant. The minimum elastic energy density, initially transferred to the lattice atoms to obtain the final vacancy distribution, is plotted as "Elastic (Displacements)". It is calculated by multiplying the vacancy distribution of silicon and carbon by their respective displacement energies of $35 \mathrm{eV}$ and $20 \mathrm{eV}$, which are the averaged minimum energies necessary to create a stable Frenkel defect, i.e. to separate the vacancy and the dislodged atom far enough to prevent spontaneous recombination and emission of a phonon. The amorphization energy is obtained from this plot by multiplying the displacement energy density at the depth of the a/c-interface with the fluence and normalizing it to the atomic target density. In a similar way the amorphization dose is calculated from the vacancy density. The initial energy of the dislodged atom is lost along its trajectory by inelastic collisions and phonon excitations and the possible creation of secondary recoils. The total elastic energy transferred to the lattice is the sum of the phonon energy and binding energy stored in vacancies. The latter is obtained by multiplying the vacancy distribution by the binding energy of $2 \mathrm{eV}$ and is plotted as "Elastic (Vacancies)" in Fig.4. 


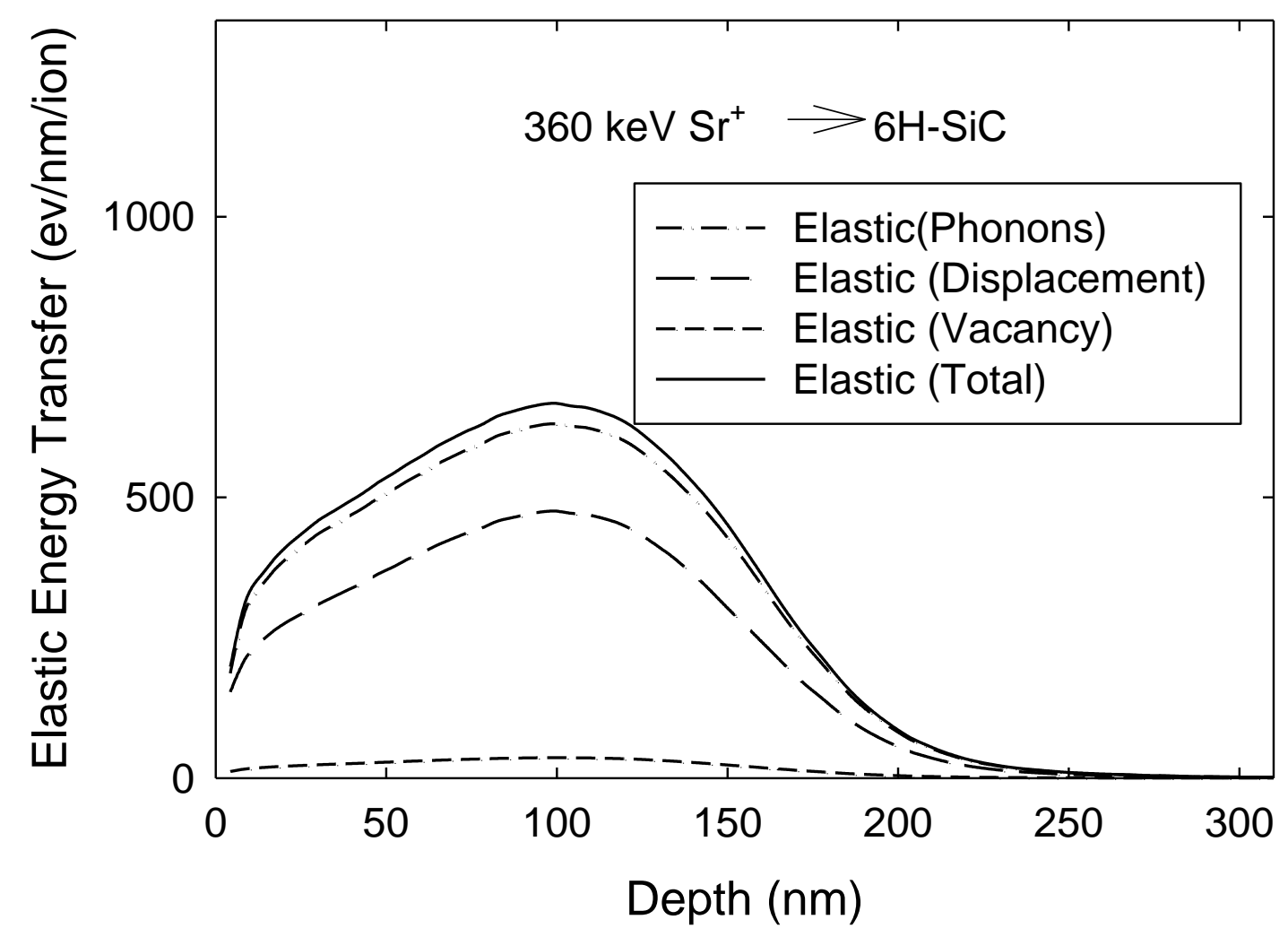

Fig. 4: TRIM simulation of elastic energy transfers for $360 \mathrm{keV} \mathrm{Sr}{ }^{+}$ions in $6 \mathrm{H}-\mathrm{SiC}$. The energy scale is adjusted by a factor of 1.019 to correct for the difference of the simulated and experimental projected ranges.

Figure 1

\section{Results and discussion}

Results for the six investigated ion types are summarized in Table 1. The amorphization energy in column 5 is the elastic energy transferred at the a/c-interface without the phonon energy to make it comparable with the amorphization dose tabulated in column 6 . The phonon energy is assumed to be locally dissipated within picoseconds as thermal energy, reducing damage efficiency by defect annealing. However, as can be judged from Fig. 4, the phonon energies are like the inelastic energy transfers relatively small at the a/c-interface and should therefore not influence significantly damage efficiencies.

Table 1: Experimental values of projected range, depth of the a/c-interface and the relative atomic density of the implanted impurity at this depth. The last two columns are obtained from TRIM simulations by multiplying the corresponding elastic energy and vacancy densities at the a/c-interface with the fluence and normalize them relative to the atomic density of SiC.

\begin{tabular}{|l|l|l|l|l|l|}
\hline Ion & $\begin{array}{l}\mathrm{R}_{\mathrm{p}} \\
(\mathrm{nm})\end{array}$ & $\begin{array}{l}\text { Depth }(\mathrm{a} / \mathrm{c}) \\
(\mathrm{nm})\end{array}$ & $\rho(\mathrm{a} / \mathrm{c})$ & $\begin{array}{l}\text { Amorphization } \\
\text { energy } \\
(\mathrm{eV} / \text { atom })\end{array}$ & $\begin{array}{l}\text { Amorphization } \\
\text { dose } \\
(\mathrm{dpa})\end{array}$ \\
\hline${ }^{84} \mathrm{Kr}$ & 139 & 241 & $1.7 \times 10^{-3}$ & 20.7 & 0.83 \\
\hline${ }^{88} \mathrm{Sr}$ & 130 & 227 & $1.7 \times 10^{-3}$ & 17.5 & 0.71 \\
\hline${ }^{107} \mathrm{Ag}$ & 109 & 220 & $0.6 \times 10^{-3}$ & 12.8 & 0.52 \\
\hline${ }^{127} \mathrm{I}$ & 97 & 193 & $0.3 \times 10^{-3}$ & 6.4 & 0.27 \\
\hline${ }^{132} \mathrm{Xe}$ & 102 & 176 & $0.4 \times 10^{-3}$ & 21.1 & 0.85 \\
\hline${ }^{133} \mathrm{Cs}$ & 106 & 179 & $0.7 \times 10^{-3}$ & 22.3 & 0.89 \\
\hline
\end{tabular}


The results for the amorphization energies and doses for silver and iodine are considerably smaller than those obtained for the other four ions. It is unlikely, that this large scatter is due to lattice stress caused by the implanted impurities, because their relative atomic densities at the a/c-interface, determined by RBS analysis and listed in column 4 of Table 1 , are all much smaller than $10^{-2}$. The observed large deviations of critical damage values are probably due to uncertainties introduced into the TRIM code by the employed approximations. Especially the use of a single universal potential applied to all binary collisions might introduce much larger errors in the tail region of the distributions, utilized in this investigation, than the generally assumed $10 \%$ uncertainty.

From the above results an average critical damage energy of $(17 \pm 6) \mathrm{eV} /$ atom and a critical dose of $(0.7 \pm 0.2)$ dpa is extracted for homogeneous amorphization of silicon carbide by low energy collisions of secondary recoils. This result agrees quite well with the critical damage energy of 18.5 eV/atom obtained for boron implantation at $180 \mathrm{keV}$ in silicon carbide at $\mathrm{z}<\mathrm{R}_{\mathrm{p}}$ [6]. In this case, a very light ion is involved for which the irradiation conditions are very similar to those reported in this paper. The low electronic stopping together with the very small probability of the occurrence of large collision cascades should lead to a homogenous amorphization process not influenced much by defect annealing, binding energy modification and non-linear effects.

Contrary to the above results, much lower amorphization doses are found for heavier ions in the near surface region of silicon carbide, where the inelastic collision density is about two orders of magnitude larger than in the region investigated in this work. Amorphization doses reported for neon, argon and xenon ions are $(0.47 \pm 0.02),(0.42 \pm 0.02)$ and $(0.35 \pm 0.02)$ dpa, respectively [8], while according to TRIM simulations the electronic stopping powers at a depth of $10 \mathrm{~nm}$ for the reported implantation parameters are $1470 \mathrm{eV} / \mathrm{nm}(\mathrm{Ne}), 1660 \mathrm{eV} / \mathrm{nm}(\mathrm{Ar})$ and $1814 \mathrm{eV} / \mathrm{nm}(\mathrm{Xe})$. Hence, less elastic energy is required to achieve complete disorder with increasing electronic stopping power. The results reveal an inverted linear relationship, which confirms the presumption previously expressed by the author [6], that the generally observed dependence of amorphization dose on the ion mass can be explained by electronic stopping. Furthermore the critical dose obtained for xenon ions near the surface is half of the value obtained far beyond the projected range, where electronic stopping is negligible. Both these results proof that inelastic energy transfer enhances damage efficiency in silicon carbide. Obviously the effect of reduced binding energy due to electronic excitation or ionization on the displacement probability of target atoms is more important than the annealing of defects. This seems to be incompatible with the results presented by Ref [8], who suggest that they indicate the dominance of an irradiation-induced recovery process. Their conclusion is based on analyses of temperature dependences of the amorphization dose at constant electronic stopping powers, which indeed are dominated by a dynamic ionization-induced recovery process. This, however, can certainly not be generalized to describe also the dependence of amorphization dose on electronic stopping power as being dominated by such a process. That this is not the case is evident from their own results, showing a decreasing amorphization dose with increasing ion mass, which means with increasing electronic stopping power as discussed above. This latter relationship cannot be explained by a recovery process. It also contradicts their introductory statement that electronic stopping power increases with decreasing ion mass.

Finally the question arises, whether the defect densities observed at the a/c-interface are not partly due to implantation related lattice stress. Although the observed impurity density at this depth is far too low to explain such an effect, shock waves created during ion stopping might well be responsible for it. Some time ago our group in cooperation with the Ion Beam Group of the Forschungszentrum Karlsruhe made an extensive investigation of deep damage beyond the projected range in metallic targets having different crystallographic structures [17]. The results showed that deep damage was linked directly to the Peierls force, which is the minimum force necessary to create a dislocation. In all targets with a face-centered cubic structure, for which the Peierls force is 
extremely low, deep damage has been observed up to a depth of about four times the projected range, while no deep damage was observed in hexagonal structures having a relatively high Peierls force. As $6 \mathrm{H}-\mathrm{SiC}$ has a double layered hexagonal structure, the possibility of deep damage created by shockwaves can therefore safely be discarded.

\section{Conclusion}

Critical amorphization values are obtained for silicon carbide implants at depths far beyond the projected range, where the influence of inelastic collisions and non-linear effects are negligible. In this depth region the results should be largely independent of the ion type and should only reflect the intrinsic properties of the target material. The observed average amorphization dose of $(0.7 \pm$ $0.2)$ dpa is significantly larger than those obtained near the surface [8], where electronic stopping is high. From this follows, that in silicon carbide the effect of binding energy reduction by electronic stopping is more important than defect annealing.

\section{Acknowledgements}

Financial support of the South African National Research Foundation is gratefully acknowledged.

\section{References}

[1] W.J. Weber, L.M. Wang, N. Yu, N.J. Hess, Mater. Sci. Eng. A253 (1998) 62.

[2] E. Wendler, A. Heft, W. Wesch, Nucl. Instr. and Meth. B 141 (1998) 105.

[3] E. Friedland, L.C. Prinsloo, Surface \& Coatings Technology 158 - 159 (2002) 64.

[4] E. Friedland, J.P.F. Sellschop, Nucl. Instr. and Meth. B 191, (2002) 17.

[5] E. Friedland, H.D. Carstanjen, G. Myburg, M.A. Nasr, Nucl. Instr. and Meth. B 230 (2005) 129.

[6] E. Friedland, Nucl. Instr. and Meth. B 217 (2004) 396.

[7] E. Friedland, Surface \& Coatings Technology 201 (2007) 8220.

[8] W.J. Weber, L. Wang, Y. Zhang, W. Jiang, I.-T. Bae, Nucl. Instr. and Meth. B 266 (2008) 2793.

[9] J.F. Ziegler, J.P. Biersack, U. Littmark, The Stopping and Ranges of Ions in Solids, Pergamon Press, New York, 1985.

[10] W,J. Weber, D.M. Duffy, L. Thome, Y. Zhang, Current Opinion in Solid State and Materials Science 19 (2015) 1.

[11] W.J. Weber, Nucl. Instr. and Meth. B 166-167, (2000) 98.

[12] E. Friedland, N.G. van der Berg, J.B. Malherbe, R.J. Kuhudzai, A.J. Botha, E. Wendler, W.Wesch, Nucl. Instr. and Meth. B 268 (2010) 2892. 
[13] K. Gärtner, Nucl. Instr. and Meth. B 227, 522 (2005).

[14] K. Gärtner, A. Ugguzoni, Nucl. Instr. and Meth. B 67, 189 (1992).

[15] E. Friedland, K. Gärtner, T.T. Hlatshwayo, N.G. van der Berg, T.T. Thabethe, Nucl. Instr. and Meth. B 332, 415 (2014).

[16] L.L. Snead, T.Nozawa, Y. Katoh, T.-S. Byun, S. Kondo, D.A. Petty, J. Nucl. Mater. 371, 329 (2007).

[17] E. Friedland, N.G. van der Berg, J. Hanßmann, O. Meyer, Surface \& Coatings Technology 83 (1996) 10. 